Monatsschrift f. Geburtshülfe u. Gynäkologie 1927;76:I-IV

\title{
Contents, Vol. 76, 1927
}

Inhaltsverzeichnis.

Or $1 / 8$ inalarbeiíen.

$1 / 8 \quad$ Seit $\theta$

A sch, R., Zur Sekaleverordnung 397

At zero dt, Kurt, Ovarialsarkom und Peritonealtuberkulose . 282

Bähr, Schweige- und Zeugnispflicht des Arztes 296

Beau camp, E., Abdominalschwangerschaft bei lebendem Kincle 2 fii0 Biehle, Hildegard, 1st der Gewichtsverlust am Ende der

Schwangerschaft ein Zeichenfiir die bevorstehendeEntbindung? 107 Brügelmann, Clara,

Beobachtungen bei Sepsis puerperalis,

insbesondere über Lokalisation und Häufigkeit der Metastasen 404

Bültemann, Hans, Einiges über Kastratoide 429

Bykow, S. G., Yersuche mit Uterusüberpflanzung in das Omen-

tum majus bei Hunden (autotransplantatio uteri) ... . 186 Ebstein, Erich, Zur Verwendung des Handschuhes in der Geburtshilfe im Jahre 1758

Esser, A., Pyosalpinx mit Leberabszeß und Hirnabszessen . 21 F r a n k 1, 0 s k a r, Zur

Kenntnis der entzündlichen Veränderungen

in Myomen 27

Fraymann, S., Über einige toxische und mechanische Ursachen

der Sehstörung bei Schwangerschaft und. Geburt .... 165 Goldschmidt, A. und J. Koerner, Zur

Prognose der Genital-

sarkome $\quad 443$

Haba, Anton, Bemerkungen zu H. I⿳1⁄8ankens Arbeit: „Über die

Beurteilung der Plazenta bezüglich ihrer Vollständigkeit, durch

eine Luftprobe statt der Milchprobe" 1

Hartmann, Heinz, Der Einfiuß entzüncllicher Adnexerkran-

kungen auf den Ablauf des mensuellen Zyklus $\quad 419$

Harttung, Zur Behandlung der Mastitis mit Eigenblut, Barden-

heuerschem Schnitt und Sekundärnaht

4

Heimann, Fritz, Nabelschnurtorsion , 255

Heyer,Eugen, Adnexentzündung während der Schwangerschaft 243 Hille, Karl, Zur Frage der Vaginalplastik nach Schubert . 288 Hinselmann, Hans, Das klinische Bild einer Plattenepithelinsel in der Zervikalschleimhaut. (Hierzu Tafel I) . 442

Hinterstoisser, Hermann, Beiträge zur Mj'omoperation . 130 Klaften, E., Über Hämagglutination und ihrepraktische Bewertung 91 Kok, Fr., Über die Ursache der Sterilität und Tubargravidität auf Grund neuerer Untersuchungen über die Tubenfunktion;

zugleich ein Beitrag zur Divertikelfrage 32

Koerner, J., siehe Goldschmidt, H. 
Kupferberg, H., Zur Therapie beim engen Becken. . . . 176

Lahm, W., Die Differenzierungshemmung der Tubenschleimhaut

und ihre Bedeutung für die Tubenpathologie. Ein Beitrag auch

für die Entstehung der Hydrosalpinx 192

Lebedeff, Valentin, Eine Operation zurBildung einer künst-

lichen Scheide aus einem Dünndarm. (Nach Mori.)... 294 Liegner,B., Intrauterin entstandene

Drucknekrosen an beiden

Unterarmen

278

Martin, Ed., Das „Schädeltrauma”

XV Inhaltsverzeichnis.

Seit»

Martin, Ed., „Avertin” (E. 107) in der Geburtshilfe . ...

- Die Levatorschenkel in der Geburt $\quad 400$

Müller, Aug., Die Wirkung der Lues der Mutter auf das Kind 216

0 den thai, Wilh., Die Behandlung des Scheidenverschlusses 122

Pulvermacher, D., Leitsätze zum Vortrag: Sorge für Mutter

und Kind. (Tagung des Vereins zur Förderung des Hebammen-

wesens, Juni 1927.)

230

R,osenstein, Walter, Über Zervixrisse bei spontanen Geburten

Schneider, Georg Heinrich, Beobachtung einer seltenen

Schwangerschaftstoxikose: Astmopathia gravidarum. . . . 115

Schroder, Hans, Über einen Fall von Fistula cervico-vagi-

nalis laqueatica 274

Seidemann, Hans, Primäre Vulva- und Scheidenkarzinome 452

Siegmund, Hermann, Diagnostische Irrtümer bei Doppel-

mißbildungen der Gebärmutter

10

Walther, Heinr., Richtlinien zur Aufhahme und Fortbildung

der Hebammen 228

Weill, L., Entwicklungsmechanik der Zelle

Vereins- und Literaturbeîlage. Original-Sitzungsberichte aus geburtshilflich-gynäkologischen

Gesellschaften:

Gesellschaft für Geburtshilfe und Gynäkologie zu Berlin.

Sitzungen vom 15. X., 29. X., 12. XL, 26. XI. und 10. XII. 192641 Sitzungen vom 14. I., 28. L, 11. II. und 25. II. $1927 \ldots 318$

Gynäkologische Gesellschaft zu 'Breslau.

Sitzung vom 16. XI. $1926 \quad 54$

Sitzung vom 25. I. $1927 \quad 308$

Sitzung vom 15.11.1927 457

Münchner Gynäkologische Gesellschaft.

Sitzung vom 18. XL $1926 \quad 63$

Sitzungen vom 14. V., 16. VII., 26. XL 1925, 28. I. 1926.137

Nordostdeutsche Gesellschaft für Gynäkologie.

Sitzung vom 17. VII. $1926 \quad 68$

Sitzung vom 27. XL $1926 \quad \cdot 345$

Mittelrheinische Gesellschaft.

71. Sitzung vom 12. XII. 1926 
Bayerische Gesellschaft für Geburtshilfe und Frauenheilkunde.

Sitzung vom 6. XII. $1925 \quad 349$

Geburtshilflich-gynäkologische Gesellschaft in Wien.

Sitzungen vom 9. XL u. 14. XII. $1926 \quad 375$

Sitzungen vom 11. I., 8. III., 5. IV. und 10. V. 1927 ... 463

Tagung der Deutschen Gesellschaft für Chirurgie. Berlin, 20. bis 23. IV. 1927305

Buchbesprechungen 75, 155, 377, 468

Literaturverzeichnís . .84, 157, 232, 382, 470

Personalien . 90, 164, 240, 386, 482

Druckfehlerberichtigung 240

Max Hofmeíer $\dagger \quad 240,387$

Fritz Hítschmann $\dagger \quad 478$ 